\title{
Quinone-catalyzed oxidative deformylation: synthesis of imines from amino alcohols
}

\author{
Xinyun Liu, Johnny H. Phan, Benjamin J. Haugeberg, Shrikant S. Londhe \\ and Michael D. Clift ${ }^{*}$
}

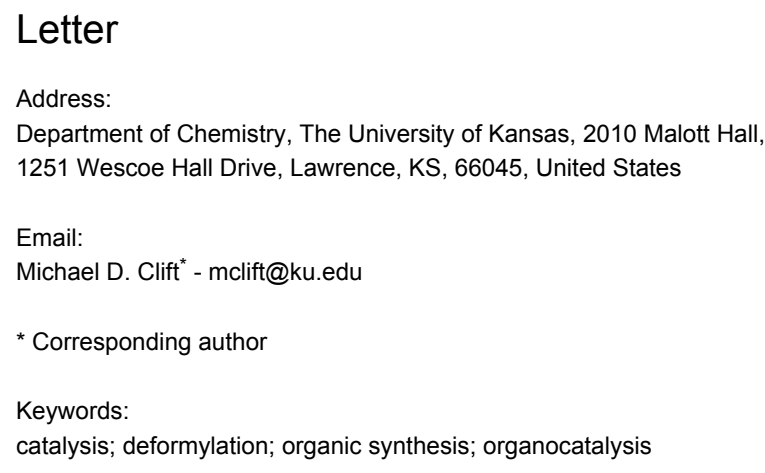

Beilstein J. Org. Chem. 2017, 13, 2895-2901.

doi:10.3762/bjoc. 13.282

Received: 21 September 2017

Accepted: 17 December 2017

Published: 28 December 2017

Associate Editor: M. Rueping

(c) 2017 Liu et al.; licensee Beilstein-Institut. License and terms: see end of document.

\begin{abstract}
A new method for imine synthesis by way of quinone-catalyzed oxidative deformylation of 1,2-amino alcohols is reported. A wide range of readily accessible amino alcohols and primary amines can be reacted to provide N-protected imine products. The methodology presented provides a novel organocatalytic approach for imine synthesis and demonstrates the synthetic versatility of quinone-catalyzed oxidative $\mathrm{C}-\mathrm{C}$ bond cleavage.
\end{abstract}

\section{Introduction}

Imines are extremely versatile intermediates in organic chemistry [1-3]. Consequently, many synthetic methods have been developed for the preparation of imines (Scheme 1). The condensation of an amine with an aldehyde or ketone is the oldest and most commonly employed method for imine synthesis [4]. More recently, the catalytic dehydrogenation of amines mediated by metal and organic catalysts has begun to emerge as an alternative approach for the preparation of imines $[5,6]$. The majority of these methods involve cleavage of a $\mathrm{C}-\mathrm{H}$ bond at the $\alpha$-position of an amine substrate [7-28]. Methods that deliver imines through amine $\alpha-\mathrm{C}-\mathrm{C}$ bond cleavage are far less common [29-32] despite the fact that these methods employ renewable resources, such as amino acids and their derivatives, as starting materials. In fact, only a few reports describing the oxidative deformylation of amino alcohols have been published [33-35], and in all of these reports stoichiometric oxidants, such as $\mathrm{NaIO}_{4}$ and $\mathrm{Pb}(\mathrm{OAc})_{4}$, must be employed to enable the desired transformations. Given that 1,2-amino alcohols are readily accessible from feedstock chemicals such as styrenes [36-38] and amino acids [39], the development of a new methodology to transform these materials into high-value imine products under catalytic conditions has the potential to be broadly useful. Herein, we report a new method that utilizes quinone catalysis to enable the synthesis of imines via oxidative deformylation of amino alcohols.

Our group has recently reported the quinone-catalyzed decarboxylative homologation of $\alpha$-amino acids [32], which 
classical methods for imine preparation - condensation (ref. [4])

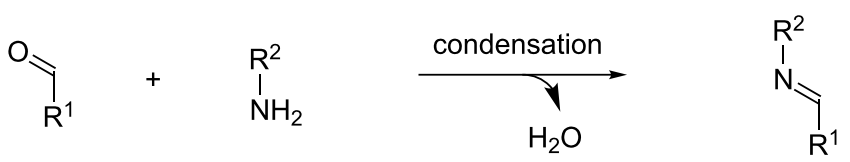

emerging methods for imine preparation - amine oxidation via $\mathrm{C}-\mathrm{H}$ bond cleavage (ref. [7-31]): $\quad$ via $\mathrm{C}-\mathrm{C}$ bond cleavage (ref. [32]):

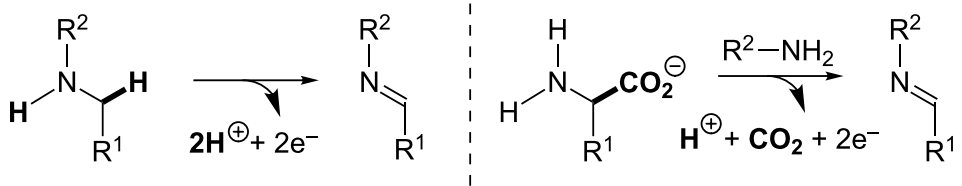

this report - imine synthesis via quinone-catalyzed deformylation<smiles>[R]C(N)C([2H])O</smiles>

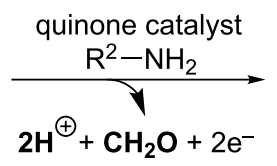<smiles>[R]C=N[R]</smiles><smiles>CC(C)(C)C1=CC(=O)C=C(C(C)(C)C)C1=O</smiles>

Scheme 1: Established methods for the preparation of imines vs this work.

demonstrated for the first time that quinone organocatalysts can be utilized to enable oxidative $\mathrm{C}-\mathrm{C}$ bond cleavage to provide versatile imine intermediates. To further exploit the utility of this chemistry, we sought to develop a new method for the preparation of a wide range of imine products through the quinonecatalyzed deformylation of 1,2-amino alcohols. Such a transformation would not only facilitate rapid access to a variety of
$\mathrm{N}$-protected imines, but would also provide a novel approach for utilizing feedstock chemicals for the preparation of these valuable synthetic intermediates.

We envisioned a process wherein a 1,2-amino alcohol 1 would undergo condensation with an appropriate quinone catalyst $\mathbf{2}$ to deliver iminoquinone 3 (Scheme 2). Deformylation of $\mathbf{3}$ would

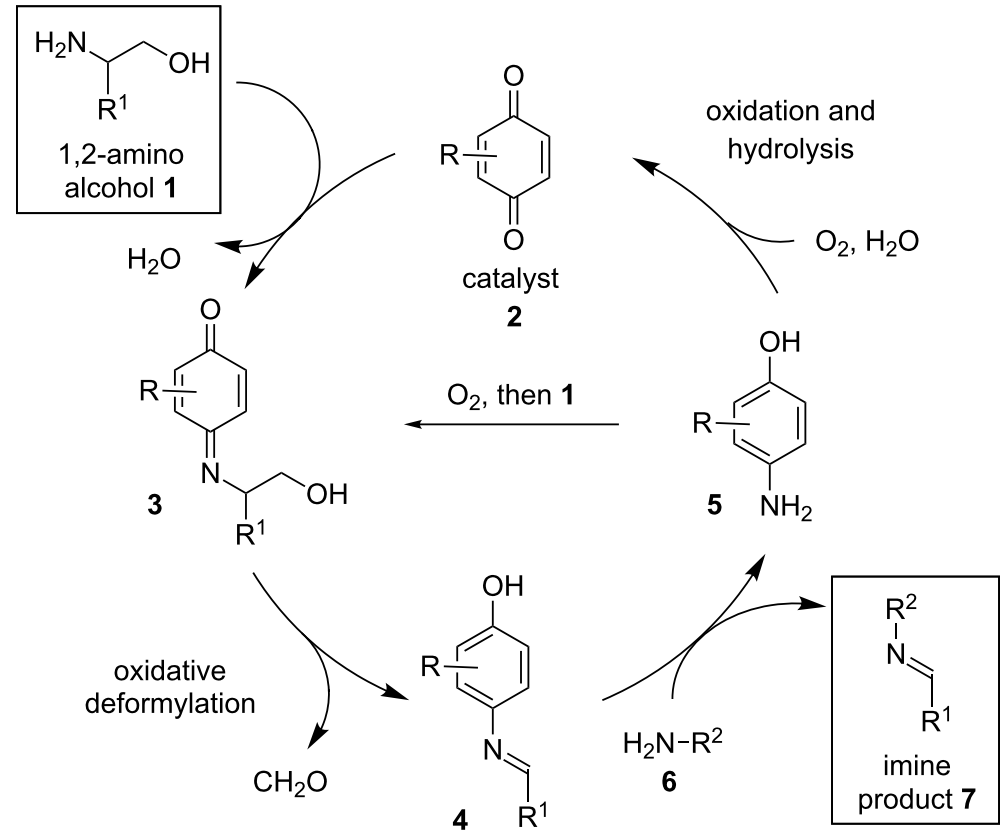

Scheme 2: Proposed catalytic cycle for quinone-catalyzed deformylation. 
generate $N$-arylimine 4. Subsequent transimination with amine 6 would provide the desired imine product 7 and a reduced form of the catalyst 5 , which would be expected to undergo oxidative turnover through one of two possible mechanisms (i.e., $5 \rightarrow 3$ or $5 \rightarrow 2$ ).

\section{Results and Discussion}

With this plan in mind, we first explored the ability of several quinone catalysts to promote the deformylation of 2-phenylglycinol (1a) to deliver $N$-PMP imine 7a (Table 1). We selected quinone catalysts $(\mathbf{2 a}-\mathbf{c})$ that have previously been utilized in amine oxidation reactions $[21,32,40,41]$, and began with reaction conditions similar to those developed for our quinone-catalyzed oxidative decarboxylation chemistry [32]. To our delight, the desired deformylation product $7 \mathbf{a}$ was formed in $63 \%$ yield when catalyst 2a was employed (Table 1, entry 1). Quinone 2b failed to deliver imine $7 \mathbf{a}$ (Table 1, entry 2), but commercially available quinone $\mathbf{2 c}$ provided $\mathbf{7 a}$ in a promising $59 \%$ yield (Table 1, entry 3). Next, we examined the effect of base on the reaction using quinone $2 \mathrm{c}$ as the catalyst (Table 1, entries 4-7). Unfortunately, no improvement in reaction efficiency was observed when different bases were employed (Table 1, entries $4-6,0-55 \%$ yield); however, exclusion of the base provided imine 7a in good yield (Table 1, entry 7, 85\%). Decreasing the loading of catalyst $\mathbf{2 c}$ under these conditions reduced the yield of imine $7 \mathbf{a}$ (Table 1, entry $8,64 \%$ yield), as did changing the identity of the catalyst (Table 1, entries 9 and 10, 62\% and $0 \%$ respectively). Finally, we examined a range of solvents in an effort to further improve efficiency (Table 1, entries 11-17). No improvements in reaction efficiency were observed (Table 1 , $0-72 \%$ yield), but it was noted that polar, protic solvents are critical in enabling the efficient deformylation of phenylglycinol.

With optimized conditions in hand, we next explored the scope of this methodology by employing a range of 1,2-amino alcohol substrates 1 (Table 2). As reported in Table 1, the reaction involving phenylglycinol gave the desired $N$-PMP imine (7a) in $85 \%$ yield (Table 2, entry 1). ortho-Substitution of the arene is reasonably well-tolerated, as 2-methylphenylglycinol (1b) and 2-chlorophenylglycinol (1c) delivered the corresponding imines in $68 \%$ yield (Table 2, entries 2 and 3). The meta-fluoro derivative provided imine $7 \mathbf{d}$ in $60 \%$ yield (Table 2 , entry 4 ). Electronic effects were studied by examining a series of para-substituted phenylglycinol derivatives (Table 2, entries 5-9). Both electron-donating and electron-withdrawing substituents were tolerated, but no obvious trends in the reactivity patterns were observed (47-77\% yield). Thiophenyl amino alcohol $\mathbf{1} \mathbf{j}$ was also subjected to the optimized conditions and the corresponding imine $\mathbf{7} \mathbf{j}$ was formed in $47 \%$ yield (Table 2, entry 10 ). Unfortunately, aliphatic 1,2-amino alcohols, such as valinol
Table 1: Optimization of quinone-catalyzed oxidative deformylation of phenylglycinol (1a)

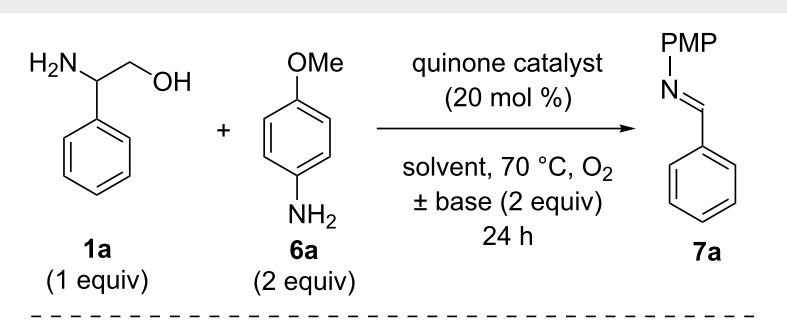

quinone catalysts tested:

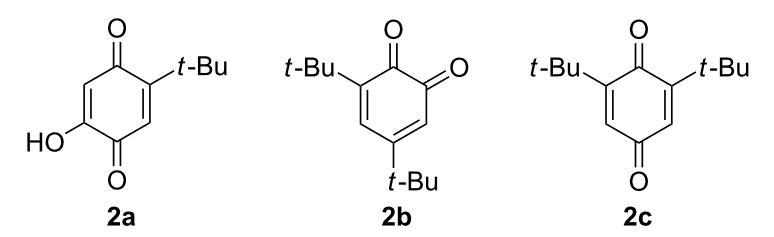

\begin{tabular}{lllll}
\hline Entry & Catalyst & Solvent & Base & Yield [\%] \\
\hline 1 & 2a & $\mathrm{EtOH}$ & $\mathrm{Et}_{3} \mathrm{~N}$ & 63 \\
2 & 2b & $\mathrm{EtOH}$ & $\mathrm{Et}_{3} \mathrm{~N}$ & 0 \\
3 & 2c & $\mathrm{EtOH}$ & $\mathrm{Et}_{3} \mathrm{~N}$ & 59 \\
4 & 2c & $\mathrm{EtOH}$ & $\mathrm{DABCO}$ & 55 \\
5 & 2c & $\mathrm{EtOH}$ & $\mathrm{DBU}$ & 0 \\
6 & 2c & $\mathrm{EtOH}$ & $\mathrm{K}_{2} \mathrm{CO}$ & 17 \\
7 & 2c & $\mathrm{EtOH}$ & none & 85 \\
$8^{\mathrm{b}}$ & 2c & $\mathrm{EtOH}$ & none & 64 \\
9 & 2a & $\mathrm{EtOH}$ & none & 62 \\
10 & 2b & $\mathrm{EtOH}^{2}$ & none & 0 \\
11 & 2c & $\mathrm{iPrOH}$ & none & 72 \\
12 & 2c & $\mathrm{H}_{2} \mathrm{O}$ & none & 47 \\
13 & 2c & $\mathrm{MeCN}$ & none & 28 \\
14 & 2c & $\mathrm{DMSO}$ & none & 13 \\
$15^{\mathrm{c}}$ & 2c & $\mathrm{THF}$ & none & 0 \\
16 & 2c & $\mathrm{PhMe}$ & none & 11 \\
17 & 2c & $\mathrm{CHCl} 3$ & none & 3 \\
\hline
\end{tabular}

aDetermined by ${ }^{1} \mathrm{H}$ NMR using benzyl ether as an internal standard. b10 mol \% quinone was used.

${ }^{\mathrm{C}}$ Reaction carried out at $50^{\circ} \mathrm{C}$.

(1k), failed to undergo deformylation under the current conditions (Table 2, entry 11).

Next, we investigated the use of various amine reaction partners 6 to access a variety of imine products 7 from phenylglycinol (1a, Table 3). The reaction with aniline (61, Table 3, entry $2,68 \%$ yield) showed reduced reaction efficiency compared to that with para-anisidine (6a, Table 3, entry 1, 85\% yield). When para-fluoroaniline (6m) was employed as the reaction partner, imine $\mathbf{7 m}$ was produced in a $77 \%$ yield (Table 3 , entry 3). $\alpha$-Branched amines are effective reaction partners, providing the corresponding imines $\mathbf{7} \mathbf{n}-\mathbf{p}$ in modest yields (Table 3 , entries $4-6,42-66 \%$ yield). From these results, it can be concluded that increasing the steric bulk at the $\alpha$-position of the 
Table 2: Quinone-catalyzed oxidative deformylation of various amino alcohols.<smiles>[R]=CC=CC(N)C(N)CO</smiles>

$6 a$

1

(1 equiv)

(2 equiv) quinone catalyst $2 \mathrm{c}$

(20 mol \%)

$\mathrm{EtOH}, 70^{\circ} \mathrm{C}, \mathrm{O}_{2}$ $24 \mathrm{~h}$

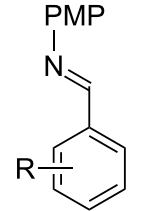

7<smiles>CC(C)(C)C1=CC(=O)C=C(C(C)(C)C)C1=O</smiles>

2c

\begin{tabular}{llll}
\hline Entry & Amino alcohol 1 & Product 7 & Yield [\%] $^{\mathrm{a}}$ \\
\hline
\end{tabular}

4<smiles>[R]c1ccccc1C(N)CO</smiles><smiles>NC(CO)c1cccc(F)c1</smiles><smiles>[R]c1ccc(C(N)CO)cc1</smiles><smiles>NC(CO)c1cccs1</smiles>

$1 \mathbf{j}$

ee, $\mathrm{R}=\mathrm{Me}$

1f, $\mathrm{R}=\mathrm{OMe}$

1g, $\mathrm{R}=\mathrm{Cl}$

1h, $R=F$

1i, $\mathrm{R}=\mathrm{CF}_{3}$

1d<smiles>[R16]N=Cc1ccccc1[R]</smiles><smiles>N#[PH]/N=C/c1cccc(F)c1</smiles>

$7 d$

$7 a, R=H$

$7 \mathrm{~b}, \mathrm{R}=\mathrm{Me}$

7c, $\mathrm{R}=\mathrm{Cl}$

85

68<smiles>[R]c1ccc(/C=N/P)cc1</smiles>

$\begin{array}{ll}\text { 7e, } R=M e & 68 \\ \text { 7f, } R=O M e & 77 \\ 7 \mathbf{g}, R=C l & 66 \\ 7 \mathbf{h}, R=F & 54 \\ 7 \mathbf{i}, R=\mathrm{CF}_{3} & 47\end{array}$

7j

47<smiles>CC(=O)N=Cc1cccs1</smiles>

PMP

11<smiles>CC(C)C(N)CO</smiles>

$1 k$

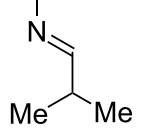

$7 k$

0

${ }^{\text {aD }}$ etermined by ${ }^{1} \mathrm{H}$ NMR using benzyl ether as an internal standard (average of two replicates).

amine results in decreased reaction efficiency. Phenethylamine (6q) provided only a $17 \%$ yield of the corresponding imine (7q, Table 3, entry 7), potentially due to its increased nucleophilicity, which may result in inhibition of catalysis via condensation with quinone 2c. We also tested several electron deficient amides $(\mathbf{6 r}-\mathbf{t})$ in these reactions (Table 3, entries 8-10). Unfortunately, only sulfinamide $\mathbf{6 t}$ provided the desired imine $\mathbf{7 t}$ (Table 3, entry 10, 22\% yield). In all three cases, a significant amount of benzaldehyde was observed, indicating that electron deficient primary amides (such as $\mathbf{6 r}-\mathbf{t}$ ) are either incapable of promoting transimination, or the resulting imines $(\mathbf{7} \mathbf{r}-\mathbf{t})$ are hydrolyzed under the current reaction conditions. Notably, imines 7n [42-45] and 7t [46-48] are useful imines for diastereoselective 1,2-addition reactions.

Following these substrate scope studies, we next examined the quinone-catalyzed $\mathrm{C}-\mathrm{C}$ bond cleavage of analogous substrates (Scheme 3). First, we tested isomeric amino alcohol iso-1a, which provided imine $\mathbf{7 a}$ in a yield comparable to that observed when phenylglycinol was used as a substrate. Notably, the mechanism of this reaction likely involves initial formation of benzaldehyde, followed by condensation with para-anisidine, 
Table 3: Quinone-catalyzed oxidative deformylation using various amine reaction partners.<smiles>NC(CO)c1ccccc1</smiles>

$1 \mathrm{a}$

(1 equiv)

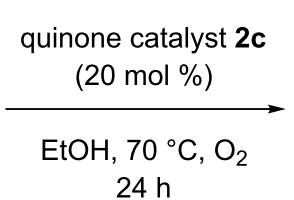

$24 \mathrm{~h}$

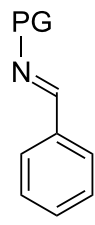

7<smiles>O=C1C=CC(=CBr)C(=O)C([18Br])=C1</smiles>

2c

\begin{tabular}{lll}
\hline Entry & Amine 6 & Product 7
\end{tabular}

1

2<smiles>CC(N)c1ccccc1</smiles>

$6 n$

6a, $\mathrm{R}=\mathrm{OMe}$

6I, $\mathrm{R}=\mathrm{H}$

$6 \mathrm{~m}, \mathrm{R}=\mathrm{F}$<smiles>NC(c1ccccc1)c1ccccc1</smiles>

60<smiles>CC(C)(C)N</smiles>

$6 p$<smiles>NCCc1ccccc1</smiles>

$6 q$<smiles>[13CH3]OC(N)=O</smiles>

$6 r$<smiles></smiles>

$6 s$

$6 t$<smiles>[R]c1ccc(/N=C/c2ccccc2)cc1</smiles><smiles>CC(/N=C/c1ccccc1)c1ccccc1</smiles>

$7 n$

66<smiles>C(=N/C(c1ccccc1)c1ccccc1)\c1ccccc1</smiles>

70

42<smiles>CC(C)(C)N=Cc1ccccc1</smiles>

$7 \mathrm{a}, \mathrm{R}=\mathrm{OMe}$

7I, $\mathrm{R}=\mathrm{H}$

$7 \mathrm{~m}, \mathrm{R}=\mathrm{F}$

68

77

56<smiles>C(=N/CCc1ccccc1)\Pc1ccccc1</smiles>

$7 q$<smiles>[13CH3]OC(=O)/N=C/P</smiles>

$7 r$

0

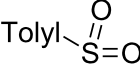<smiles>C=Nc1ccccc1</smiles>

$7 s$

$t-\mathrm{Bu}_{\mathrm{s}_{\mathrm{s}}}=\mathrm{O}$

$N>\quad 7 t$

22

aDetermined by ${ }^{1} \mathrm{H}$ NMR using benzyl ether as an internal standard (average of two replicates).

to deliver imine 7a. Vicinal diamine $\mathbf{8}$ was also a compatible substrate, delivering imine $\mathbf{7 a}$ in $63 \%$ yield. Finally, we subjected diol 9 to the optimal reaction conditions; no product was ob- served, indicating that condensation between the substrate and catalyst to form an iminoquinone intermediate is likely required for productive reactivity. 


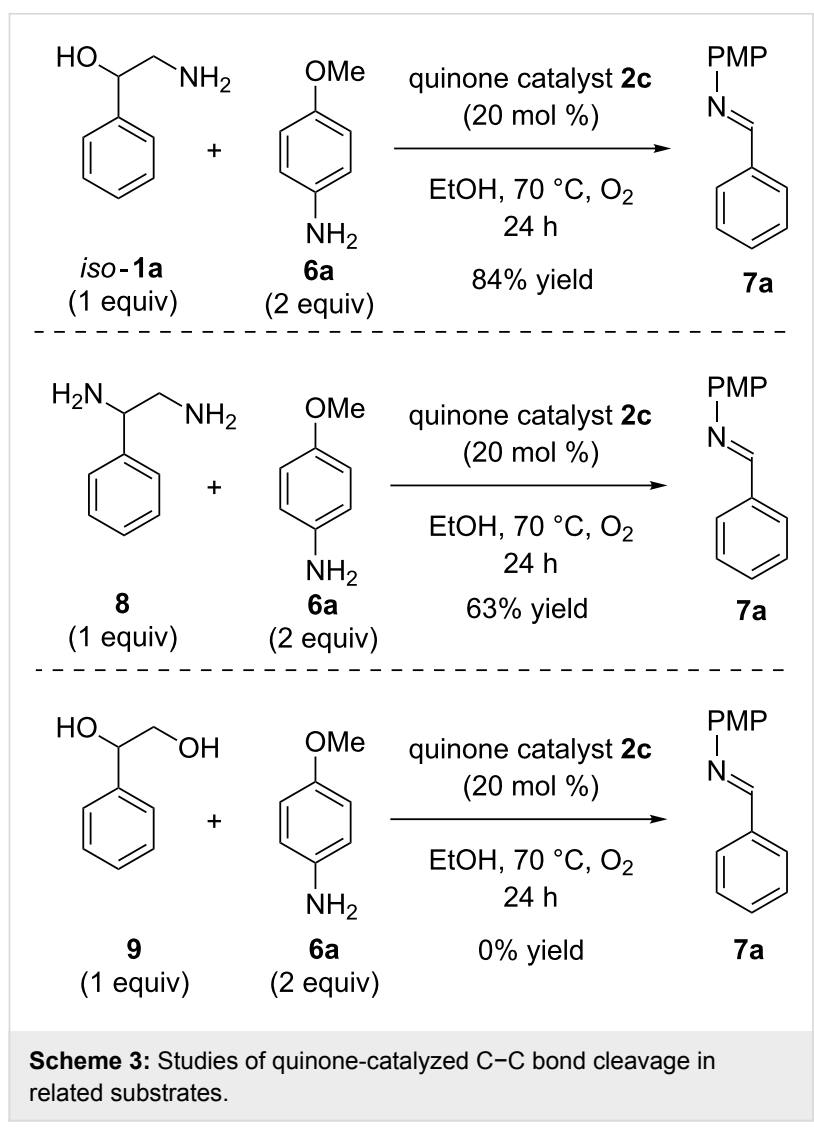

To demonstrate the synthetic utility of this methodology, we performed a sequential oxidative deformylation/ Mukaiyama-Mannich addition under our previously reported conditions for decarboxylative amino acid homologation (Scheme 4) [32]. In this reaction sequence, (thio)silyl ketene acetal $\mathbf{1 0}$ was united with 2-phenylglycinol and para-anisidine in a two-step, one-pot process to provide $\beta$-amino acid derivative 11 in a $60 \%$ yield. The overall reaction sequence provides a unique method for the production of the high-value $\beta$-amino acid derivatives $[49,50]$ from 1,2-amino alcohols.

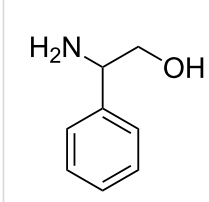

$1 \mathrm{a}$

(1 equiv)

\section{2 c, $(20 \mathrm{~mol} \%)$, EtOH} $p$-anisidine ( 2 equiv) $70^{\circ} \mathrm{C}, \mathrm{O}_{2}, 24 \mathrm{~h}$

2. $\mathrm{HBF}_{4}, \mathrm{THF}, 0^{\circ} \mathrm{C}, 2 \mathrm{~h}$

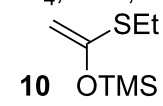

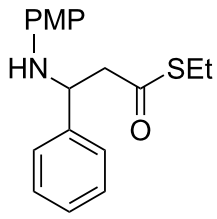

11 $60 \%$ yield
Scheme 4: Sequential oxidative deformylation/Mukaiyama-Mannich addition using phenylglycinol.

\section{Conclusion}

In conclusion, we have developed a novel method for the synthesis of imines from 1,2-amino alcohols. This chemistry fea- tures an unprecedented application of quinone organocatalysis to enable oxidative deformylation under aerobic conditions. Future work will involve mechanistic studies and the development of new catalysts to expand the scope of this chemistry.

\section{Supporting Information}

\section{Supporting Information File 1}

Experimental procedures, compound characterization data, and copies of ${ }^{1} \mathrm{H}$ and ${ }^{13} \mathrm{C}$ NMR spectra.

[http://www.beilstein-journals.org/bjoc/content/ supplementary/1860-5397-13-282-S1.pdf]

\section{Acknowledgements}

Financial support from the NSF (EPS-0993806) and The University of Kansas is gratefully acknowledged. Additional support for this work was provided by the National Institutes of Health Graduate Training Program in Dynamic Aspects of Chemical Biology Grant T32 GM08545 from NIGMS (to B. J. H.). Support for NMR instrumentation was provided by NIH Shared Instrumentation Grants No. S10OD016369 and S10RR024664, and NSF Major Research Instrumentation Grant No. 0320648 .

\section{ORCID ${ }^{\circledR}$ iDs}

Michael D. Clift - https://orcid.org/0000-0001-6441-9802

\section{References}

1. Kobayashi, S.; Ishitani, H. Chem. Rev. 1999, 99, 1069-1094. doi:10.1021/cr980414z

2. Adams, J. P. J. Chem. Soc., Perkin Trans. 1 2000, 125-139. doi:10.1039/a808142e

3. Kobayashi, S.; Mori, Y.; Fossey, J. S.; Salter, M. M. Chem. Rev. 2011, 111, 2626-2704. doi:10.1021/cr100204f

4. Schiff, H. Justus Liebigs Ann. Chem. 1864, 131, 118-119. doi:10.1002/jlac.18641310113

5. Patil, R. D.; Adimurthy, S. Asian J. Org. Chem. 2013, 2, 726-744. doi:10.1002/ajoc.201300012

6. Chen, B.; Wang, L.; Gao, S. ACS Catal. 2015, 5, 5851-5876. doi:10.1021/acscatal.5b01479

7. Éll, A. H.; Samec, J. S. M.; Brasse, C.; Bäckvall, J.-E. Chem. Commun. 2002, 1144-1145. doi:10.1039/b202117j

8. Murahashi, S.-I.; Okano, Y.; Sato, H.; Nakae, T.; Komiya, N. Synlett 2007, 1675-1678. doi:10.1055/s-2007-984515

9. Zhu, B.; Angelici, R. J. Chem. Commun. 2007, 21, 2157-2159. doi:10.1039/b700555e

10. Jiang, G.; Chen, J.; Huang, J.-S.; Che, C.-M. Org. Lett. 2009, 11, 4568-4571. doi:10.1021/ol9018166

11. Allen, J. M.; Lambert, T. H. J. Am. Chem. Soc. 2011, 133, 1260-1262. doi:10.1021/ja109617y

12. Patil, R. D.; Adimurthy, S. Adv. Synth. Catal. 2011, 353, 1695-1700. doi:10.1002/adsc. 201100100

13. Prades, A.; Peris, E.; Albrecht, M. Organometallics 2011, 30 , 1162-1167. doi:10.1021/om101145y 
14. Rueping, M.; Zhu, S.; Koenigs, R. M. Chem. Commun. 2011, 47, 12709-12711. doi:10.1039/c1cc15643h

15. Rueping, M.; Zhu, S.; Koenigs, R. M. Chem. Commun. 2011, 47, 8679-8681. doi:10.1039/c1cc12907d

16. Freeman, D. B.; Furst, L.; Condie, A. G.; Stephenson, C. R. J. Org. Lett. 2012, 14, 94-97. doi:10.1021/ol202883v

17. Largeron, M.; Fleury, M.-B. Angew. Chem., Int. Ed. 2012, 51, 5409-5412. doi:10.1002/anie.201200587

18. Park, J. H.; Ko, K. C.; Kim, E.; Park, N.; Ko, J. H.; Ryu, D. H.; Ahn, T. K.; Lee, J. Y.; Son, S. U. Org. Lett. 2012, 14, 5502-5505. doi:10.1021/ol302584y

19. Rueping, M.; Koenigs, R. M.; Poscharny, K.; Fabry, D. C.; Leonori, D.; Vila, C. Chem. - Eur. J. 2012, 18, 5170-5174. doi:10.1002/chem.201200050

20. Rueping, M.; Zoller, J.; Fabry, D. C.; Poscharny, K.; Koenigs, R. M.; Weirich, T. E.; Mayer, J. Chem. - Eur. J. 2012, 18, 3478-3481. doi:10.1002/chem.201103242

21. Wendlandt, A. E.; Stahl, S. S. Org. Lett. 2012, 14, 2850-2853. doi:10.1021/ol301095j

22. Zhu, S.; Rueping, M. Chem. Commun. 2012, 48, 11960-11962. doi:10.1039/c2cc36995h

23. Zhang, E.; Tian, H.; Xu, S.; Yu, X.; Xu, Q. Org. Lett. 2013, 15 , 2704-2707. doi:10.1021/ol4010118

24. Bergonzini, G.; Schindler, C. S.; Wallentin, C.-J.; Jacobsen, E. N.; Stephenson, C. R. J. Chem. Sci. 2014, 5, 112-116. doi:10.1039/C3SC52265B

25. Furukawa, S.; Suga, A.; Komatsu, T. Chem. Commun. 2014, 50, 3277-3280. doi:10.1039/C4CC00024B

26. Wang, J.; Lu, S.; Cao, X.; Gu, H. Chem. Commun. 2014, 50 , 5637-5640. doi:10.1039/c4cc01389a

27. Wendlandt, A. E.; Stahl, S. S. J. Am. Chem. Soc. 2014, 136, 506-512. doi:10.1021/ja411692v

28. Beatty, J. W.; Stephenson, C. R. J. Acc. Chem. Res. 2015, 48, 1474-1484. doi:10.1021/acs.accounts.5b00068

29. Leonard, N. J.; Rebenstorf, M. A. J. Am. Chem. Soc. 1945, 67, 49-51. doi:10.1021/ja01217a016

30. McGarvey, G. J.; Williams, J. M.; Hiner, R. N.; Matsubara, Y.; Oh, T. J. Am. Chem. Soc. 1986, 108, 4943-4952. doi:10.1021/ja00276a040

31. Shimizu, M.; Makino, H. Tetrahedron Lett. 2001, 42, 8865-8868. doi:10.1016/S0040-4039(01)01945-1

32. Haugeberg, B. J.; Phan, J. H.; Liu, X.; O'Connor, T. J.; Clift, M. D. Chem. Commun. 2017, 53, 3062-3065. doi:10.1039/C7CC00485K

33. Burger, E. C.; Tunge, J. A. J. Am. Chem. Soc. 2006, 128, 10002-10003. doi:10.1021/ja063115x

34. Zuo, Z.; MacMillan, D. W. C. J. Am. Chem. Soc. 2014, 136, 5257-5260. doi:10.1021/ja501621q

35. Zuo, Z.; Cong, H.; Li, W.; Choi, J.; Fu, G. C.; MacMillan, D. W. C. J. Am. Chem. Soc. 2016, 138, 1832-1835. doi:10.1021/jacs.5b13211

36. Reddy, K. L.; Dress, K. R.; Sharpless, K. B. Tetrahedron Lett. 1998, 39 , 3667-3670. doi:10.1016/S0040-4039(98)00644-3

37. Reddy, K. L.; Sharpless, K. B. J. Am. Chem. Soc. 1998, 120, 1207-1217. doi:10.1021/ja9728177

38. Williamson, K. S.; Yoon, T. P. J. Am. Chem. Soc. 2010, 132, 4570-4571. doi:10.1021/ja1013536

39. Nystrom, R. F.; Brown, W. G. J. Am. Chem. Soc. 1947, 69, 2548-2549. doi:10.1021/ja01202a082

40. Corey, E. J.; Achiwa, K. J. Am. Chem. Soc. 1969, 91, 1429-1432. doi:10.1021/ja01034a027

41. Leon, M. A.; Liu, X.; Phan, J. H.; Clift, M. D. Eur. J. Org. Chem. 2016, 4508-4515. doi:10.1002/ejoc.201600786
42. Boga, C.; Savoia, D.; Umani-Ronchi, A. Tetrahedron: Asymmetry 1990 1, 291-294. doi:10.1016/S0957-4166(00)86316-8

43. Annunziata, R.; Benaglia, M.; Cinquini, M.; Cozzi, F.; Raimondi, L. Tetrahedron Lett. 1998, 39, 3333-3336. doi:10.1016/S0040-4039(98)00484-5

44. Nancy; Ghosh, S.; Singh, N.; Nanda, G. K.; Venugopalan, P.; Bharatam, P. V.; Trehan, S. Chem. Commun. 2003, 1420-1421. doi:10.1039/b300478c

45. Chen, J.; Pandey, R. K.; Cunico, R. F. Tetrahedron: Asymmetry 2005, 16, 941-947. doi:10.1016/j.tetasy.2005.01.025

46. Ellman, J. A.; Owens, T. D.; Tang, T. P. Acc. Chem. Res. 2002, 35, 984-995. doi:10.1021/ar020066u

47. Ellman, J. A. Pure Appl. Chem. 2003, 75, 39-46. doi:10.1351/pac200375010039

48. Robak, M. T.; Herbage, M. A.; Ellman, J. A. Chem. Rev. 2010, 110, 3600-3740. doi:10.1021/cr900382t

49. Cheng, R. P.; Gellman, S. H.; DeGrado, W. F. Chem. Rev. 2001, 101, 3219-3232. doi:10.1021/cr000045i

50. Weiner, B.; Szymański, W.; Janssen, D. B.; Minnaard, A. J.; Feringa, B. L. Chem. Soc. Rev. 2010, 39, 1656-1691. doi:10.1039/b919599h

\section{License and Terms}

This is an Open Access article under the terms of the Creative Commons Attribution License (http://creativecommons.org/licenses/by/4.0), which permits unrestricted use, distribution, and reproduction in any medium, provided the original work is properly cited.

The license is subject to the Beilstein Journal of Organic Chemistry terms and conditions:

(http://www.beilstein-journals.org/bjoc)

The definitive version of this article is the electronic one which can be found at: doi:10.3762/bjoc. 13.282 